\title{
Análise de Métodos de Deteç̧ão de Vazamento com Foco em Operações de Offloading
}

\begin{abstract}
Rodrigo P. A. Rejaili
Resumo - A pesquisa foi realizada para obter-se uma comparação entre diferentes sensores, avaliando a aplicabilidade desses na detecção e mitigação de vazamentos na operação de Offloading (transferência de óleo entre a plataforma e a FPSO). Para tal, avaliou-se diferentes métodos de detecção de vazamento em água, óleo e gás quanto a instalação, manutenção, precisão, entre outros aspectos, definindo-se pesos para cada um deles. A partir disso, cada método recebeu uma nota, que possibilitou a escolha da solução mais adequada.
\end{abstract}

Palavras-chave - vazamento; detecção; mitigação; offloading; FPSO; óleo; gás; água

\section{Introdução}

A operação de offloading é realizada para transferir-se o óleo da plataforma de prospecção para o FPSO (navio aliviador), o qual transporta o óleo até a terra. Tal operação é realizada diversas vezes, sendo que a mesma pode durar 24 horas ou mais. Dado todo esse tempo de operação, um pequeno vazamento pode despejar vários litros de óleo no mar, causando diversos prejuízos ao meio ambiente. Com isso, percebeu-se a importância do desenvolvimento de um método de detecção e mitigação do vazamento para reduzir ao máximo a quantidade de óleo perdida, e, portanto, o impacto ambiental causado. Portanto, realizou-se uma comparação entre diferentes métodos de detecção de vazamento a fim de investigar sua aplicabilidade em operações de offloading.

\section{Métodos estudados}

Os métodos de detecção analisados na pesquisa foram retirados de artigos científicos, selecionados os de maior relevância e com maior número de citações, e procurando-se métodos de detecção e mitigação de vazamentosem tubulações de óleo, gás e água. Uma vez selecionados os artigos, os mesmos foram caracterizados por uma série de aspectos considerados pertinentes à detecção de vazamento (COLOMBAROLI, 2009), acrescentando-se outros relevantes na operação de offloading. Por último, compilaram-se todos os métodos analisados em uma tabela, para a melhor comparação entre os mesmos, atribuindo-se notas a cada um dos aspectos de cada método.

\subsection{Inteligência Artificial (SUKARNO et al., 2007)}

A pesquisa foi desenvolvida utilizando-se dados de vazamento hipotéticos em um oleoduto reto de $80 \mathrm{~km}$ e 24 " de diâmetro externo com taxa de vazamento de $1 \%$. O modelo de oleoduto simula o fluxo de óleo no duto e prediz a pressão ao longo do duto com ou sem vazamento, em uma certa condição de fluxo. O vazamento é representado por uma ramificação do duto, com certo diâmetro, e que pode estar localizada em qualquer lugar do duto. Assim, com a taxa de vazamento, a distribuição de pressão ao longo do duto é calculada.

Para se analisar a presença ou não de vazamentos, precisa-se saber a pressão de entrada e saída do duto. $\mathrm{O}$ modelo de detecção de vazamentos depende de formulação matemática e do ANN (Artificial Neural Network, método que replica a inteligência humana, podendo reconhecer padrões em certos dados). Baseado nos resultados do modelo de oleoduto e do modelamento matemático, o software para prever os problemas de vazamentos é desenvolvido. O software consiste em um modelo de detecção de vazamentos, e sub-rotina que contém dados de vazamentos hipotéticos em 
dutos que foram gerados usando o modelo de oleoduto. O mesmo identifica, por meio de um método iterativo, com os dados de pressão de entrada e saída, a presença e a localização de vazamentos.

As vantagens e limitações que puderam ser retiradas do artigo foram compiladas nas tabelas I e II.

Tabela I Vantagens do método de inteligência artificial

\begin{tabular}{cc}
\hline \hline $\begin{array}{c}\text { Funcionamento sob } \\
\text { mudanças operacionais }\end{array}$ & $\begin{array}{c}\text { Os dados de entrada devem cobrir os casos onde se deseja a detecção, } \\
\text { possibilitando a detecção para diferentes diâmetros e comprimentos de } \\
\text { dutos, pressões de entrada e saída e taxas de fluxo. }\end{array}$ \\
\hline \hline
\end{tabular}

Tabela II Limitações do método de inteligência artificial

\begin{tabular}{cc}
\hline \hline Precisão de detecção & $\begin{array}{c}\text { Depende do número de iterações realizadas, exigindo maior tempo. Não } \\
\text { alcançou boa precisão nos resultados. }\end{array}$ \\
\hline Disponibilidade 24h & $\begin{array}{r}\text { Para uma precisão razoável do local do vazamento seriam necessárias } \\
20.000 \text { iterações, o que toma 1 hora de computação }\end{array}$ \\
\hline $\begin{array}{c}\text { Instalação e } \\
\text { Manutenção }\end{array}$ & $\begin{array}{c}\text { Quanto maior a complexidade do duto, maior o tempo necessário para } \\
\text { preparar-se os dados de treinamento do software. }\end{array}$ \\
\hline $\begin{array}{c}\text { Aderência ao uso em } \\
\text { offloading }\end{array}$ & $\begin{array}{c}\text { Como o mangote não é rígido, o método provavelmente não será eficaz, } \\
\text { uma vez que o método exige configuração fixa. O tempo de resposta com } \\
\text { boa precisão prejudica a utilização na mitigação. }\end{array}$ \\
\hline \hline
\end{tabular}

\subsection{Baseados em Transiente (COLOMBO et al., 2009)}

As análises foram realizadas com métodos de detecção de vazamentos em sistemas de distribuição de água. Os diferentes métodos abordados estão a seguir.

\section{A Características Gerais}

O comportamento hidráulico do sistema de tubulações é explorado em tais métodos. Qualquer mudança na estrutura física ou propagação da tubulação como uma junção, constrição, expansão, bloqueio, transição de dureza ou vazamento impõe uma reflexão de onda para um sinal de transiente, alterando o fluxo do sistema e sua resposta de pressão.

\section{B Análise de Transiente Inverso}

Nesse método, o estado do sistema é conhecido/calculado (pressões, algumas demandas, fluxos, etc.) mas alguns parâmetros (dureza da tubulação, outras demandas, vazamentos, etc.) são desconhecidos. Tipicamente, as variáveis de estado do sistema são gravadas durante um evento transiente e comparadas com medições correspondentes ao mesmo sistema livre de vazamentos. Singularidades potenciais são então testadas em um simulador hidráulico-numérico até que os traços de pressão do sistema com e sem vazamentos estejam satisfatoriamente combinados. Então, o problema inverso é resolvido para os parâmetros de sistema como dureza da tubulação e tamanho e posição do vazamento.

\section{Técnicas de Domínio de Frequência}

Tais técnicas usualmente envolvem a medição do histórico de pressão em apenas uma seção de medição. Elas também envolvem um dispositivo periodicamente atuado, geralmente uma válvula controlada para a geração de transiente e análise da resposta da frequência do sistema durante o fluxo estável-oscilatório resultante. Uma vez que tal fluxo é estabelecido em um sistema, as medições de pressão são feitas e as amplitudes máximas são determinadas para cada frequência, gerando um diagrama da resposta da frequência, o qual pode gerar comparações entre sistemas intactos e aqueles com singularidades como vazamentos e quebras. 


\section{Análise de Transiente Direto - Reflectometria por Domínio de Tempo}

A técnica foi testada em tubulações de emissários (outfall pipes). $\mathrm{O}$ estudo considerou um único cano sujeito a um transiente iniciado na extremidade de montante. Quando um sinal propagado de transiente encontra um vazamento, parte da energia da onda principal é desviada para formar um novo sinal refletido. A detecção desse sinal refletido, e a medição do tempo de chegada do mesmo, podem ser aproveitados para expor o vazamento.

\section{E Análise de Transiente Direto - Amortecimento de Transiente}

Tal método explora a propriedade de atenuação da pressão causada pelos vazamentos.

As vantagens e limitações globais desse método se encontram nas tabelas III e IV.

Tabela III Vantagem dos métodos baseados em transiente

\begin{tabular}{cc}
\hline $\begin{array}{c}\text { Disponibilidade } \\
24 \mathrm{~h}\end{array}$ & Grande potencial de ser utilizado em um sistema de detecção de vazamentos \\
em tempo real
\end{tabular}

Tabela IV Limitações dos métodos baseados em transiente

\begin{tabular}{cc}
\hline \hline $\begin{array}{c}\text { Funcionamento sob } \\
\text { mudanças operacionais }\end{array}$ & $\begin{array}{c}\text { Os ruídos do sistema, que em um sistema complexo podem ser } \\
\text { originados por diversas fontes, dificultam a separação dos sinais de } \\
\text { vazamento com outras perturbações e eventos }\end{array}$ \\
\hline $\begin{array}{c}\text { Custo de implantação / } \\
\text { Instalação e Manutenção }\end{array}$ & $\begin{array}{c}\text { Tal método de detecção de vazamentos ainda não é largamente } \\
\text { conhecido e aplicado na prática, por ser um método relativamente } \\
\text { novo }\end{array}$ \\
\hline \hline
\end{tabular}

\subsection{Resposta por Frequência (SATTAR e CHAUDHRY, 2008)}

Uma onda transiente é gerada na extremidade a jusante de um oleoduto por uma válvula e se propaga até o vazamento, onde é refletida. A onda refletida, que depende da descarga do vazamento e da localização deste, volta para a válvula trazendo informações sobre o vazamento. Após a repetição deste processo de reflexão, pode-se detectar a localização do vazamento interpretando a frequência de todo o sistema. Verifica-se que um vazamento em um duto modifica a forma da frequência, aumentando a amplitude da pressão de oscilação. Este aumento na amplitude ocorre de modo a determinar um padrão oscilatório ao longo do eixo da frequência, de modo que a frequência da resposta da onda e sua amplitude estão diretamente relacionadas com a localização e descarga do vazamento.

Por meio desta técnica não é necessário conhecer a pressão transitória anterior ao vazamento, bastando conhecer o transiente em algum ponto do duto. Isto torna esta técnica mais vantajosa que muitas outras, uma vez que é mais simples de ser usada e do ponto de vista teórico é rápida, eficiente e econômica.

A coluna dorsal deste método é a detecção da resposta por frequência obtida pelo método frequency sweep technique, logo uma extração apurada desta resposta é essencial para o sucesso deste método. Os vazamentos foram analisados em dutos demasiadamente simples, desconsiderando algumas condições que são comuns em sistemas industriais, tais como ramificações, loops, ou outras fronteiras. Por conseguinte, há uma limitação na obtenção da resposta por frequência em situações mais complexas. Propõe-se, para estas situações, que o sistema seja analisado em subsistemas mais simples. Para vazamentos múltiplos as reflexões das ondas de pressão resultarão em um padrão mais complexo para a frequência, sendo necessária uma descrição mais adequada para este ponto.

As vantagens e limitações desse método estão descritas nas tabelas V e VI. 
Tabela V Vantagens do método de resposta por frequência

\begin{tabular}{|c|c|}
\hline $\begin{array}{l}\text { Sensibilidade a } \\
\text { vazamentos }\end{array}$ & $\begin{array}{l}\text { Em sistemas simples, vazamentos tão baixos quanto } 0,2 \% \text { do fluxo de } \\
\text { descarga são detectados }\end{array}$ \\
\hline $\begin{array}{c}\text { Estimativa de } \\
\text { localização }\end{array}$ & $\begin{array}{l}\text { Excelente, no entanto é necessário um grande número de dados para } \\
\text { obter-se boa acurácia }\end{array}$ \\
\hline
\end{tabular}

Tabela VI Limitação do método de resposta por frequência

Funcionamento sob mudanças

operacionais
Não garante o funcionamento em diferentes

operações

\subsection{Acústico (MENG et al., 2011)}

Propõe-se um método acústico de detecção de vazamento. Para isto, implementa-se um método capaz de distinguir ondas acústicas de vazamentos de outros tipos de sinais, como ruído de fundo e sinais de distorção, provocados por ligamento e desligamento de compressores, abertura de válvulas, choques no duto, etc. Estes sinais de distorção são parecidos com o sinal acústico do vazamento.

Posicionam-se dois sensores de detecção de ondas acústicas nas extremidades do duto. Quando ocorre um vazamento, ondas acústicas são geradas por meio do atrito com as paredes do duto. Os sinais acústicos são recebidos e tratados como ruído de fundo. Quando ocorre um vazamento, o sinal acústico de vazamento e o ruído de fundo chegam ao sensor e são comparados com os perfis de sinais de ondas acústicas presentes no sistema. O método permite a detecção de vazamentos analisando componentes de baixa frequência do sinal sonoro observado pelos sensores. A localização do vazamento é feita analisando a velocidade da onda sonora e da diferença de tempo que cada sensor detecta o sinal.

O experimento realizado com este método de detecção de vazamento foi testado sob um diversas pressões entre 1,6 e 4,6 MPa. O comprimento total do duto utilizado é 251,5 m e possui um diâmetro interno de $10 \mathrm{~mm}$. O comprimento usado para teste no duto foi de $200,8 \mathrm{~m}$. O número de Reynolds considerado foi maior que 107, o que é consistente para transmissões em dutos. O gás sai do tanque de alta pressão e passa para a zona de teste, posteriormente vai para um tanque de média pressão e, então, é descartado.

A tabela VII mostra as vantagens do método.

Tabela VII Vantagens do método acústico

\begin{tabular}{cc}
\hline $\begin{array}{c}\text { Sensibilidade a } \\
\text { vazamentos }\end{array}$ & $\begin{array}{c}\text { Vazamentos com aberturas acima de 0,45 mm e pressão abaixo de 1,6 MPa não } \\
\text { foram detectados (erros elevados para pequenas pressões/aberturas) }\end{array}$ \\
\hline $\begin{array}{c}\text { Estimativa de } \\
\text { localização }\end{array}$ & O erro máximo diagnosticado foi de 1,37\% e o erro mínimo foi de 0,01\%. \\
\hline $\begin{array}{c}\text { Taxa de alarmes } \\
\text { falsos }\end{array}$ & $\begin{array}{c}\text { O sinal acústico de um vazamento é comparado com sinais externos no domínio } \\
\text { do tempo, que contêm forma de onda, amplitude, valor quadrático médio (valor } \\
\text { eficaz), curtose, assimetria, etc. Se estes dados puderem ser usados juntos, a } \\
\text { taxa de alarme falso é drasticamente reduzida. }\end{array}$ \\
\hline \hline
\end{tabular}

\subsection{Sensores de Fibra Óptica (TANIMOLA e HILL, 2009)}

O método em questão consiste na análise da reflexão característica de pulsos de laser emitidos através de um cabo de fibra ótica. Sensores instalados a cada metro ao longo do cabo medem as alterações na reflexão do laser ao longo do cabo. Essas medições são feitas analisando a intensidade e a cor dos pulsos de laser. Essas alterações são consequências de mudanças da temperatura, de surgimento de tensões e de efeitos de vibrações ao longo do cabo de fibra ótica. Diferentes tipos de sensores são instalados para interpretar diferentes tipos de alterações no cabo.

Detecção por sensores DTS (Distributed Temperature Sensors): Uma fenda ou um vazamento no duto causa uma liberação de fluido para a atmosfera. O cabo é instalado próximo à tubulação de 
forma que o contato do fluido com o cabo de fibra ótica gera uma queda na temperatura deste e essa diferença é detectada pelo sistema de sensores DTS.

Detecção por sensores DAS (Distributed Acoustic Sensing): Ocorrendo vibrações e perturbações no cabo, há alteração na forma como a luz reflete. Um Coherent Optical Domain Reflectometer (C-OTDR) pode detectar essa alteração.

A aplicação do método foi feita em 3 casos. O primeiro é em uma tubulação a céu aberto de $4,1 \mathrm{~km}$ de comprimento e $36 "$ de diâmetro para transporte de gás natural liquefeito entre duas estações. O segundo é uma tubulação enterrada de $57 \mathrm{~km}$ de comprimento para transporte de gás etileno. O terceiro caso foi um experimento de detecção de interferências no escoamento geradas por intrusos na tubulação e com a fibra ótica colocada a $1,2 \mathrm{~m}$ da tubulação.

As vantagens e limitações do método estão nas tabelas VIII e IX.

Tabela VIII Vantagens do método de fibra óptica

\begin{tabular}{cc}
\hline \hline Estimativa de localização & Erros de localização entre um e dois metros \\
\hline Tempo de resposta & Abaixo de dez segundos \\
\hline Disponibilidade $24 \mathrm{~h}$ & Sim \\
\hline Taxa de alarmes falsos & Eficiente na redução de alarmes falsos \\
\hline Instalação e Manutenção & Simples, sensores utilizados não são intrusivos \\
\hline Custo de implantação & Econômico e com excelente retorno de investimentos \\
\hline \hline
\end{tabular}

Tabela IX Limitação do método de fibra óptica

Instrumentação necessária Sensores necessários a cada metro de tubulação

\subsection{Transmissão de pressão dinâmica (ZHANG et al., 2013)}

Dois sensores de mudança de pressão de baixa frequência (Dynamic Pressure Transmitter - DPT) são instalados nas estações de entrada e saída da tubulação. O DPT é composto por transdutor piezoelétrico, amplificador de carga, módulo de condicionamento de sinal e um microcontrolador. O microcontrolador é utilizado para controlar o condicionamento de sinais e para converter sinais analógicos para digitais. A tubulação estudada transporta óleo, possui 94,21 quilômetros de comprimento e 457 milímetros de diâmetro.

$\mathrm{O}$ vazamento gera uma mudança de pressão que se traduz em $0,02 \mathrm{~V}$. Utiliza-se um método matemático denominado WPE (Wavelet Package Entropy) para extrair dados de pressão dinâmica dos sinais transientes de baixa frequência. $\mathrm{O}$ cálculo da posição do vazamento é feito através da velocidade de propagação da onda de pressão no fluido e a diferença de tempo de detecção entre os sinais. Foram obtidos 3 tipos de sinais de pressão dinâmica: (i) sinal de procedimento normal, (ii) sinal de vazamento e (iii) sinal de operações.

As vantagens do uso desse método estão compiladas na tabela $X$.

Tabela X Vantagens do método de transmissão de pressão dinâmica

\begin{tabular}{|c|c|}
\hline Precisão de detecção & Detecção de $96,7 \%$ da ocorrência de vazamentos \\
\hline Estimativa de localização & Precisão ótima (erro máximo de $0,134 \%$ ) \\
\hline $\begin{array}{l}\text { Funcionamento sob mudanças } \\
\text { operacionais }\end{array}$ & Diferencia sinais de operação e vazamento \\
\hline Disponibilidade $24 \mathrm{~h}$ & Tempo ilimitado \\
\hline Taxa de alarmes falsos & Baixa \\
\hline Instalação e Manutenção & $\begin{array}{l}\text { Não exige alta especialização, utiliza algoritmos simples e } \\
\text { computação de baixa complexidade }\end{array}$ \\
\hline Custo de implantação & Baixo \\
\hline
\end{tabular}




\subsection{Sensor Óptico (MCSTAY et al., 2007)}

O método óptico de detecção de vazamento de fluidos em tubulações submersas é baseado na fluorescência do fluido. Dois veículos não tripulados são usados no método: a unidade de excitação, que emite luz num comprimento de onda controlado e excita as moléculas florescentes, e a unidade de detecção, que coleta a luz emitida no mesmo comprimento de onda que processa as informações e repassa para o sistema central de controle.

O método foi estudado sob o pretexto da necessidade de se desenvolver métodos rápidos, eficientes e com longo alcance, a fim de se reduzir o tempo de inspeção e os custos, e de permitir as ROVs e AUVs manterem distância em relação à estrutura inspecionada, o que, por sua vez, garante mais segurança, evitando manobras críticas para desviar das estruturas inspecionadas.

Pode-se esperar algumas dificuldades no uso do método relacionadas ao ambiente marinho já instável, como variações de pressão e temperatura, exposição à água salgada e areia, ocorrência de corrosão e de movimentos tectônicos. As vantagens e limitações do método estão nas tabelas XI e XII.

Tabela XI Vantagens do método com sensor óptico

\begin{tabular}{cc}
\hline \hline Sensibilidade a vazamentos & Concentrações na ordem de partículas por milhão (ppm) \\
\hline Tempo de resposta & Baixo \\
\hline Taxa de alarmes falsos & Existe a possibilidade de averiguação de suspeita de vazamento \\
\hline \hline
\end{tabular}

Tabela XII Limitações do método com sensor óptico

\begin{tabular}{cc}
\hline \hline Disponibilidade 24h & Depende da disponibilidade do veículo não tripulado em operação \\
\hline $\begin{array}{c}\text { Custo de implantação / } \\
\text { Instalação e Manutenção }\end{array}$ & $\begin{array}{c}\text { Envolve veículos não tripulados com led de emissão de luz, filtro de } \\
\text { polarização da luz, detector da luz e processamento de sinal e o } \\
\text { sistema central de processamento de informações }\end{array}$ \\
\hline \hline
\end{tabular}

\subsection{Fusão de Informações de Processo (ZHANG et al., 2008)}

Este sistema de detecção de vazamentos foi estabelecido na tubulação West Products Pipeline de $1858 \mathrm{~km}$ de comprimento. A tubulação transporta óleo pelo método de bombeamento de linha justa (tight line pumping), e todas as bombas, instrumentação na linha e tubulação formam um sistema hidráulico uniforme. A tubulação passa por terreno extremamente montanhoso e zona desértica. A elevação vai de $350 \mathrm{~m}$ a $2827 \mathrm{~m}$. A tubulação tem $559 \mathrm{~mm}$ de diâmetro da estação de Wulumuqi até a estação de Ximpu, e $508 \mathrm{~mm}$ de Ximpu até Lanzhou. Existem doze estações de bombeamento ao longo da tubulação, seis estações de medida e separação de transporte, uma estação de medida e enchimento de óleo, cinquenta estações de válvula de bloqueio e cinco estações de válvulas de alto alívio. Na estação de bombeamento de Wulumuqi, existem medidores ultra-sônicos de fluxo a jusante e a montante, medidores de pressão e de temperatura. Nas outras estações de bombeamento, existem medidores de pressão a jusante e montante, e medidores de temperatura e ultra-sônico de fluxo a montante. Em todas as estações de bloqueio, existem todos os medidores das outras de bombeamento, exceto pelo ultra-sônico de fluxo a montante, que está disponível nas cinco válvulas de bloqueio. Em todas as estações de válvulas de alto alívio existem medidores de pressão a montante.

O sistema de detecção de vazamentos é baseado no método de fusão de informações de processo. O sistema coleta as informações de diferentes sensores em diferentes nós do sistema distribuído de coleta de dados com frequência de coleta diferente. É mais importante a coleta de informações com altas frequências suficientes para a detecção de vazamentos.

As vantagens e limitações do método analisado estão descritas nas tabelas XIII e XIV. 
Tabela XIII Vantagens do método de fusão de informações

\begin{tabular}{|c|c|}
\hline Precisão de detecção & Preciso e sensível na detecção a vazamentos \\
\hline Estimativa de localização & $\begin{array}{l}\text { O erro da posição no teste foi de } 2.67 \mathrm{~km} \text {, e a precisão da localização } \\
\text { da posição da fonte de flutuações foi de } 99.02 \%\end{array}$ \\
\hline $\begin{array}{l}\text { Funcionamento sob } \\
\text { mudanças operacionais }\end{array}$ & $\begin{array}{c}\text { Distingue bombas ligando, reduzindo a velocidade e parando o } \\
\text { transporte de vazamentos. Resiste a ruídos de alta frequência e baixa } \\
\text { energia. }\end{array}$ \\
\hline Taxa de alarmes falsos & Baixa \\
\hline Instalação e Manutenção & $\begin{array}{l}\text { Possui capacidade de aprendizado, ou seja, para melhorar seu } \\
\text { desempenho, os ajustes de parâmetros devem ser feitos tanto na etapa } \\
\text { de projeto quanto durante o funcionamento. }\end{array}$ \\
\hline Disponibilidade $24 \mathrm{~h}$ & Sim, não é citado, porém, o tempo de resposta \\
\hline Custo de implantação & Custo-eficiente, segundo o autor, com ressalva \\
\hline \multicolumn{2}{|c|}{ Tabela XIV Limitações do método de fusão de informações } \\
\hline $\begin{array}{l}\text { Instalação e } \\
\text { Manutenção }\end{array}$ & Exige mão de obra especializada \\
\hline $\begin{array}{l}\text { Custo de } \\
\text { implantação }\end{array}$ & Alto, dado os sensores que a tubulação possui \\
\hline $\begin{array}{cc}\text { Aderência ao uso } & \text { Limi } \\
\text { em offloading } & \text { e con }\end{array}$ & $\begin{array}{l}\text { da, pois o método é para uma tubulação grande com configuração fixa, } \\
\text { diversos sensores em locais variados, e o mangote é pequeno e flexível, } \\
\text { não sendo possível instalar muitos sensores }\end{array}$ \\
\hline
\end{tabular}

\subsection{Câmeras Infravermelho (ZHOU e ZENG, 2006)}

Trata-se de uma técnica de alinhamento de quadros de vídeo que foram levemente desalinhados (alignment of video frames), com o intuito de aumentar a eficiência no processamento automatizado de imagens. O método do alinhamento de quadros consiste na transformação de uma imagem (quadro) de forma a corresponder à outra, apresentando a maior "semelhança" possível. Este processo é chamado image registration.

O estudo é baseado na problemática da detecção de vazamento de gás e seu reparo (LDAR), no uso de equipamento de processamento de imagens de câmeras infravermelho (detecção de variações da temperatura) para vazamento de compostos orgânicos voláteis (VOC) e na possibilidade de perturbações do ambiente provocarem o desalinhamento dos quadros do vídeo no momento da filmagem.

No momento do vazamento do VOC, a rápida compressão e descompressão causam a queda da temperatura do VOC e o aumento da temperatura do ar em volta. Essa diferença de temperatura é detectada pelas câmeras de radiação infravermelha. Com o intuito de automatizar essa detecção, identificando uma nuvem de vazamento de VOC, quantificando a relação entre a imagem e a taxa de vazamento e comprimindo o vídeo para economizar espaço de armazenamento, propõe-se fazer o alinhamento dos quadros de imagem.

As tabelas XV e XVI apresentam as vantagens e limitações do uso de câmeras infravermelho na detecção de vazamento.

Tabela XV Vantagens do método de câmeras infravermelho

\begin{tabular}{|c|c|}
\hline Precisão de detecção & $\begin{array}{c}\text { Definida pela precisão do sensor e qualidade de processamento das } \\
\text { imagens }\end{array}$ \\
\hline $\begin{array}{l}\text { Taxa de alarmes } \\
\text { falsos }\end{array}$ & O alinhamento de quadros pode otimizar a análise de imagens \\
\hline Disponibilidade $24 \mathrm{~h}$ & Sim \\
\hline
\end{tabular}


Tabela XVI Limitações do método de câmeras infravermelho

Funcionamento sob

mudanças operacionais
Outras variações de temperatura, além das provocadas pelo vazamento de gás, podem ser detectadas e apontadas como um vazamento

Instalação e Manutenção

Possui certa complexidade

\subsection{Velocidade Acústica (LI et al., 2014)}

Para a detecção de vazamentos em dutos de gás com método acústico, usava-se como premissa que a velocidade do som é conhecida e invariável. Na prática a propagação de ondas sonoras no vazamento em diferentes caminhos de transmissão e nas paredes do duto apresentam diferentes velocidades do som e diferentes comportamentos de dispersão o que dificulta a detecção e localização de vazamentos. Nesse estudo, baseado na teoria de vibração e em fórmulas de número de onda foram analisadas as velocidades do som dentro do tubo e na casca para determinar o caminho de transmissão predominante. Adicionalmente a velocidade de dispersão no caminho dominante é suprimida pela seleção de uma banda de frequências característica de ondas acústicas induzidas pelo vazamento de gás.

A determinação da velocidade do som pode tornar-se complexa, pois depende das propriedades do fluido no conduto e do acoplamento acústico entre a parede e o fluido, entre outras propriedades. Necessitando, portanto, de um estudo prévio do fluido em questão e de seu comportamento dentro do conduto.

As vantagens e desvantagens desse método estão nas tabelas XVII e XVIII.

Tabela XVII Vantagens do método de velocidade acústica

\begin{tabular}{cc}
\hline \hline Precisão de detecção & Boa sensibilidade à detecção \\
\hline Estimativa de localização & Boa \\
\hline Taxa de alarmes falsos & Baixa \\
\hline Instalação e Manutenção & Uso de sensores externos ao duto \\
\hline Disponibilidade $24 \mathrm{~h}$ & Sim \\
\hline \hline
\end{tabular}

Tabela XVIII Limitações do método de velocidade acústica

\begin{tabular}{cc}
\hline \hline Instalação e Manutenção & Exige mão de obra qualificada \\
\hline Custo de implantação & Médio, depende do comprimento do duto \\
\hline $\begin{array}{c}\text { Funcionamento sob mudanças } \\
\text { operacionais }\end{array}$ & $\begin{array}{c}\text { A detecção de sinais é afetada pela interferência de ruídos } \\
\text { acústicos gerados por válvulas e compressores }\end{array}$ \\
\hline \hline
\end{tabular}

\subsection{Detecção de Hidrocarbonetos na Água (REPSOL, 2014)}

A pesquisa foi desenvolvida especificamente para detecção de hidrocarbonetos na água, de forma que esta detecção independa das condições do mar ou da visibilidade da água. A pesquisa foi realizada em laboratório reproduzindo condições climatológicas encontradas em alto mar, utilizou algoritmos de interpretação de imagens, radares, câmeras de infravermelho, centros de comando e ainda pode comunicar sua posição para evitar a colisão de outros navios.

O método combina sensores de monitoramento, interpretação automática e alarmes, permitindo a detecção sem intervenção humana e com um tempo de resposta muito pequeno. Os radares detectam diferenças de rugosidade na superfície da água quando há presença de hidrocarbonetos, enquanto a câmera de infravelho detecta variações de temperatura entre a água e o hidrocarboneto devido à diferença das propriedades térmicas. Além disso, quando o vazamento é detectado, todas as informações, registros e análises são coletadas.

As vantagens e desvantagens encontradas estão dispostas nas tabelas XIX e XX. 
Tabela XIX Vantagens do método de detecção de hidrocarbonetos na água

\begin{tabular}{cc}
\hline \hline Tempo de resposta & Inferior a dois minutos \\
\hline Taxa de alarmes falsos & Baixa \\
\hline Disponibilidade $24 \mathrm{~h}$ & Sim \\
\hline \hline
\end{tabular}

Tabela XX Limitações do método de detecção de hidrocarbonetos na água Instalação e Manutenção $\quad$ Exige mão de obra especializada

Custo de implantação Alto, devido aos sensores que a tubulação possui

\section{Resultados}

Analisados os métodos, compilou-se uma tabela comparativa geral, envolvendo todos os métodos analisados. Incluiu-se as avaliações de cada um dos aspectos avaliados, possibilitando a comparação entre eles, para a posterior classificação de acordo com a aplicabilidade na detecção e mitigação de vazamentos em operações de Offloading. A tabela compilada se encontra na figura 1.

\begin{tabular}{|c|c|c|c|c|c|c|c|c|c|c|}
\hline Método & Fluido & $\begin{array}{l}\text { Sensibilidade } \\
\text { a Vazamentos }\end{array}$ & $\begin{array}{l}\text { Precisão na } \\
\text { Localização }\end{array}$ & $\begin{array}{c}\text { Tempo de } \\
\text { resposta }\end{array}$ & $\begin{array}{c}\text { Funcionamento } \\
\text { sob mudanças } \\
\text { operacionais }\end{array}$ & \begin{tabular}{|c|} 
Disponivel \\
$24 \mathrm{~h}$
\end{tabular} & $\begin{array}{l}\text { Taxa de } \\
\text { alarmes } \\
\text { falsos }\end{array}$ & $\begin{array}{l}\text { Especialização } \\
\text { da manutenção }\end{array}$ & $\begin{array}{l}\text { Instrumentação } \\
\text { necessária }\end{array}$ & $\begin{array}{l}\text { Adequação à } \\
\text { atividade de } \\
\text { Offloading }\end{array}$ \\
\hline $\begin{array}{c}\text { Pressão c/ uso } \\
\text { de Inteligência } \\
\text { Artificial }\end{array}$ & Óleo & $\begin{array}{c}\text { Taza de } \\
\text { vazamento de } \\
1 \% \text { - Alta }\end{array}$ & \begin{tabular}{|c|} 
Erro de $62,5 \%$ \\
p/ resposta \\
rápida - Baixa
\end{tabular} & \begin{tabular}{|c|} 
Para erro \\
de $1 \%: 1$ \\
hora - Alto
\end{tabular} & Não (sem dados) & Sim & - & $\begin{array}{c}\text { Alta - envolve } \\
\text { inteligência } \\
\text { artificial }\end{array}$ & $\begin{array}{c}\text { Sensores na } \\
\text { entrada/saída e } \\
\text { inteligência - Média }\end{array}$ & Baixa \\
\hline $\begin{array}{l}\text { Baseados em } \\
\text { Transiente }\end{array}$ & Água & - & \begin{tabular}{|c|}
$\begin{array}{c}\text { Condições } \\
\text { controladas - } \\
\text { Alta }\end{array}$ \\
\end{tabular} & $\begin{array}{c}\text { Tempo real } \\
\text { - Baixo }\end{array}$ & Não & Sim & Média & \begin{tabular}{|c|} 
Alta - método \\
novo, ainda não \\
muito aplicado \\
\end{tabular} & $\begin{array}{c}\text { Vários sensores - } \\
\text { Alta }\end{array}$ & Baixa \\
\hline $\begin{array}{l}\text { Resposta por } \\
\text { Frequência }\end{array}$ & Água & $\begin{array}{c}0,2 \% \text { da vazão } \\
\text { do duto - Alta }\end{array}$ & - & - & Não & Sim & $\begin{array}{c}\text { Baixa (sem } \\
\text { dados) }\end{array}$ & Média & $\begin{array}{l}\text { Precisão cresce } \\
\text { com o número de } \\
\text { sensores - Alta }\end{array}$ & Baixa \\
\hline Acústico & Gás & $\begin{array}{c}\text { Dano com 5\% } \\
\text { do diâmetro do } \\
\text { duto - Alta }\end{array}$ & $\begin{array}{l}\text { Maior erro: } \\
1,37 \% \text { - Alta }\end{array}$ & $\begin{array}{c}\text { Instantâneo } \\
\text { - Baixo }\end{array}$ & Sim & Sim & $\begin{array}{c}\text { Baixa (sem } \\
\text { dados) }\end{array}$ & Média & $\begin{array}{c}\text { Sensores ao longo } \\
\text { do duto - Média }\end{array}$ & Baixa \\
\hline $\begin{array}{l}\text { Sensores de } \\
\text { Fibra Ótica }\end{array}$ & Óleo e Gás & - & $1 \mathrm{~m}$ a $2 \mathrm{~m}$ - Alta & $\begin{array}{c}\text { Abaixo de } \\
\text { 10s - Baixo }\end{array}$ & Sim & Sim & $\begin{array}{c}\text { Baixa (sem } \\
\text { dados) }\end{array}$ & Baixa & $\begin{array}{c}\text { Sensores a cada } \\
1 \mathrm{~m} \text { - Alta }\end{array}$ & Média \\
\hline \begin{tabular}{|c|} 
Transmissão de \\
Pressão \\
Dinâmica \\
\end{tabular} & Óleo & $96,7 \%$ - Alta & $\begin{array}{c}\text { Maior erro: } \\
0,134 \% \text { - Alta }\end{array}$ & 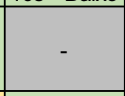 & Sim & Sim & $\begin{array}{c}\text { Baixa (sem } \\
\text { dados) }\end{array}$ & Baixa & $\begin{array}{c}\text { Sensores na } \\
\text { entrada e saída da } \\
\text { tubulação - Baixa }\end{array}$ & Média \\
\hline Sensor Óptico & Óleo e Gás & $\begin{array}{c}\text { Detecção na } \\
\text { ordem de } \\
\text { ppm/ppb - Alta }\end{array}$ & $\begin{array}{c}3 \mathrm{~m} \text { de } \\
\text { distância dos } \\
\text { dutos - Média }\end{array}$ & \begin{tabular}{|c} 
Instantâneo \\
- Baixo
\end{tabular} & - & - & Baixa & Alta & $\begin{array}{l}\text { Veículos não } \\
\text { tripulados com } \\
\text { sensores- Média }\end{array}$ & Média \\
\hline \begin{tabular}{|c|} 
Fusão de \\
informações de \\
processo
\end{tabular} & Óleo & $\begin{array}{l}\text { Alta (sem } \\
\text { dados) }\end{array}$ & \begin{tabular}{|c|}
$99.02 \%$ (erro \\
de $2.67 \mathrm{~km})$ - \\
Alta
\end{tabular} & - & Sim & Sim & $\begin{array}{c}\text { Baixa (sem } \\
\text { dados) }\end{array}$ & $\begin{array}{l}\text { Alta - envolve } \\
\text { diversos } \\
\text { sensores }\end{array}$ & \begin{tabular}{|c|} 
Diversos sensores \\
ao longo da \\
tubulação - Alta
\end{tabular} & Média \\
\hline $\begin{array}{c}\text { Câmeras } \\
\text { Infravermelho }\end{array}$ & Óleo e Gás & $\begin{array}{l}\text { Depende do } \\
\text { sensor - Alta }\end{array}$ & - & Baixo & Não & Sim & $\begin{array}{c}\text { Baixa (sem } \\
\text { dados) }\end{array}$ & Alta & $\begin{array}{c}\text { Câmeras } \\
\text { infravermelho - } \\
\text { Média } \\
\end{array}$ & Alta \\
\hline $\begin{array}{l}\text { Velocidade } \\
\text { Acústica }\end{array}$ & Gás & Alta & Alta & Baixo & $\begin{array}{c}\text { Sim (suscetível a } \\
\text { alarme falso) }\end{array}$ & Sim & \begin{tabular}{|c|} 
Média - \\
variações \\
em válvulas \\
\end{tabular} & $\begin{array}{c}\text { Baixa - software } \\
\text { e sensores }\end{array}$ & $\begin{array}{c}\text { Sensores e } \\
\text { Computador - Baixa }\end{array}$ & Alta \\
\hline \begin{tabular}{|c|} 
Detecção de \\
Hidrocarbonetos \\
na Água \\
\end{tabular} & Óleo & Alta & - & \begin{tabular}{|c|} 
Menor que \\
$2 \mathrm{~min}-$ \\
Baixo \\
\end{tabular} & Sim & Sim & Baixa & Alta & $\begin{array}{c}\text { Câmeras de } \\
\text { infravermelho e } \\
\text { radares - Alta }\end{array}$ & Alta \\
\hline
\end{tabular}

Figura 1 Comparação geral entre os métodos analisados

Percebe-se, por meio da imagem, que três métodos se mostraram com alta adequação à atividade de Offloading. Outros quatro métodos possuem média aplicabilidade.

\section{Conclusões}

O método de detecção de hidrocarbonetos na água apresenta maior potencial de aplicação na mitigação de vazamentos em Offloading, uma vez que foi desenvolvido para a detecção do vazamento de hidrocarbonetos em operações offshore, mostrando bons resultados. Os outros dois métodos (câmeras infravermelho e velocidade acústica) necessitariam de maiores pesquisas para serem adaptados ao uso no cenário analisado. 


\section{AGRADECIMENTOS}

Agradeço ao Petróleo Brasileiro S.A., em especial ao Eng. Luís José Cavadas. Agradeço também aos Prof. Dr. Diolino José dos Santos Filho, Prof. Reinaldo Squillante Júnior. Agradeço, por fim, ao PET Automação e Sistemas (PET Mecatrônica) e seus membros.

\section{REFERÊNCIAS}

COLOMBAROLI, Pedro Lucio Stefani. Sistema de Detecção de Vazamento em Dutos de Petróleo. Itajubá: Universidade Federal de Itajubá, 2009.

COLOMBO, Andrew F.; LEE, Pedro; et al. A selective literature review of transient-based leak detection methods. Journal of Hydro-environment Research, v. 2, n. 4: Elsevier B.V., 2009, p. 212-227.

LI, Shuaiyong; WEN, Yumei; et al. Determination of acoustic speed for improving leak detection and location in gas pipelines. Review of Scientific Instruments, v. 85, n. 2: AIP Publishing LLC, 2014, 11 p.

MCSTAY, D.; KERLIN, J.; et al. An optical sensor for the detection of leaks from subsea pipelines and risers. Journal of Physics: Conference Series, v. 76, n. 1: IOP Publishing Ltd, 2007, $6 \mathrm{p}$.

MENG, Lingya; YUXING, Li; et al. Experimental study on leak detection and location for gas pipeline based on acoustic method. Journal of Loss Prevention in the Process Industries, v. 25, n. 1: Elsevier Ltd., 2011, p. 90-102.

REPSOL. Repsol and Indra develop a safety system to detect hydrocarbons, the first of its kind in the world. Madri, 2014. Disponível em

http://www.repsol.com/imagenes/es_en/Heads_Repsol_Indra_eng_tcm11-675858.pdf. Acesso em 07 de março de 2016.

SATTAR, Ahmed M.; CHAUDHRY, M. Hanif. Leak detection in pipelines by frequency response method. Journal of Hydraulic Research, v. 46, n. 1: Taylor and Francis, 2008, p. 138151.

SUKARNO, Pudjo; SIDARTO, Kuntjoro Adji; et al. Leak Detection Modeling and Simulation for Oil Pipeline with Artificial Intelligence Method. Journal of Engineering and Technological Sciences, v. 39, n. 1: Institut Teknologi Bandung, 2007, p. 1-19.

TANIMOLA, Femi; HILL, David. Distributed fibre optic sensors for pipeline protection. Journal of Natural Gas Science and Engineering, v. 1, n. 4-5: Elsevier B.V., 2009, p. 134-143.

ZHANG, Lai-bin; QIN, Xian-Yong; et al. Designing a reliable leak detection system for West Products Pipeline. Journal of Loss Prevention in the Process Industries, v. 22, n. 6: Elsevier Ltd., 2009, p. 981-989.

ZHANG, Yu; CHEN, Shili; et al. Leak detection monitoring system of long distance oil pipeline based on dynamic pressure transmitter. Measurement, v. 49: Elsevier Ltd., 2014, p. 382-389.

ZHOU, Liming; ZENG, Yousheng. Automatic alignment of infrared video frames for equipment leak detection. Analytica Chimica Acta, v. 584, n. 1: Elsevier B.V., 2007, p. 223-227. 
Title: Leak Detection Methods Analysis Focusing Offloading Operations

Abstract - The research was made aiming a comparison between different sensors, evaluating their applicability in the Offloading operation (oil transfer between the platform and the FPSO) leak detection and mitigation. In order to do that, different leak detection methods, applied to water, gas and oil pipelines, were analyzed regarding their install process, maintenance, precision, etc. These criteria were weighed according to their relevance, and the methods received a score, allowing the most adequate solution to be chosen.

Keywords - leak; detection; mitigation; offloading; FPSO; oil; gas; water

\section{Rodrigo Pereira Abou Rejaili}

Graduando em Engenharia Mecatrônica pela Escola Politécnica da Universidade de São Paulo Bolsista do Programa de Educação Tutorial - PET Automação e Sistemas 